\title{
Recovering High-Order Accuracy in WENO Computations of Steady-State Hyperbolic Systems
}

\author{
Sigal Gottlieb, ${ }^{1}$ David Gottlieb, ${ }^{2}$ and Chi-Wang $\mathrm{Shu}^{2}$
}

Received June 2, 2005; accepted (in revised form) October 11, 2005; Published online February 17, 2006

\begin{abstract}
In this note we consider the application of the WENO scheme to simulations of steady-state flow in a converging diverging nozzle. We demonstrate the recovery of design accuracy through Gegenbauer postprocessing, despite the degradation of the order of accuracy for the numerical solution of the Euler equations to first-order in regions where the characteristics passed through the shock. We have shown a case in which the Gegenbauer postprocessing can recover the order of accuracy right up to the shock location. This suggests that high-order accurate information which crosses through the shock may not be irretrievably lost, and we can strive to recover it through various types of postprocessing.
\end{abstract}

KEY WORDS: WENO scheme; hyperbolic system; accuracy; postprocessing.

\section{INTRODUCTION}

It has been shown $[3,15,17]$ that approximating discontinuous solutions by high-order methods yield, in general, only first-order accuracy, because information passing through the shock along characteristics is degraded to first-order. Thus, in the region of the solution, which contains information that traveled through the shock, one can expect only first-order accuracy. Over the years, this has been an argument against the use of high-order methods for shock wave calculations. However, Lax [14] argued that, for a non-linear system, high-order information is retained by a high resolution scheme and may be extracted by postprocessing. In fact, Lax's argument indicates that more high-order information is retained in high-order

\footnotetext{
${ }^{1}$ Department of Mathematics, University of Massachusetts Dartmouth, USA. E-mail: sg@dam.brown.edu

${ }^{2}$ Division of Applied Mathematics, Brown University, Providence RI, USA. E-mails: \{dig; shu\}@dam.brown.edu
} 
solutions of non-linear systems than of linear ones, since in the non-linear case the solution operator is contractive.

All high-order methods suffer from loss of accuracy in the presence of a discontinuity. When applying spectral methods to hyperbolic PDEs which feature sharp gradients and discontinuities, the accuracy deteriorates due to the well known Gibbs phenomenon, in which global approximations of discontinuous functions converge pointwise with at most first-order accuracy. In this case the approximations are oscillatory and converge non-uniformly. Recent advances in the theory and application of spectral methods indicate that high-order information is retained in stable spectral simulations of discontinuous phenomena and can be recovered by suitable postprocessing techniques. Shu and Wong [19] used postprocessing to recover spectral accuracy, everywhere in the domain, for the discontinuous solution of the one-dimensional Burgers' equation computed using the Fourier spectral method. More recently, Gelb and Tadmor [5] used the spectral viscosity methods to approximate the solution to the Burgers' equation and to a hyperbolic system - the Euler equations of gas dynamics. Postprocessing successfully recovered design order accuracy in these examples. Although Gegenbauer postprocessing was developed and applied successfully for the spectral methods and spectral viscosity and superviscosity approximations, it has not yet been applied to finite difference methods. This is primarily because the Gegenbauer postprocessor was developed to eliminate the Gibbs phenomenon, which seemingly plays no role in essentially non-oscillatory finite difference approximations.

In this note, we present experiments in postprocessing numerical solutions of the Euler equations of gas dynamics, obtained by a fifth-order weighted essentially non-oscillatory (WENO) scheme [13, 16]. In this steady state computation, the order of accuracy is diminished in a region downstream of the shock. We demonstrate that the application of the Gegenbauer postprocessing method [6-10] recovers the design order of accuracy in the $L_{2}$ and maximum norms for steady state solutions near the shock. We note that the results are purely numerical and lack theoretical justification.

We remark that, for certain steady state problems, if the schemes and the grids are both chosen carefully, high-order accuracy may be retained up to the shock from both sides without any postprocessing, e.g. the ENO calculation in [3]. The purpose of this note is not to find the best scheme and best grids for the specific problem to retain high-order accuracy (this would be a difficult, if not impossible, task in multi-dimensions), rather it is to show that when low-order accuracy appears due to shocks, the high-order information might still reside in the numerical solution and we might be able to recover it by suitable postprocessing. 
The note is organized as follows. In Sec. 2 we describe the WENO method used to solve the steady state converging diverging nozzle problem. In Sec. 3 the Gegenbauer postprocessing is described. In Sec. 4 we present and discuss the results of the numerical experiments. In Sec. 5 we conclude and discuss future work, which will involve genuinely timedependent problems, multi-dimensional applications, different postprocessing techniques, and a search for theoretical justification of the success of the postprocessor.

\section{THE WENO METHOD}

To approximate, in a physically correct way, the solution to a conservation law of the form

$$
u_{t}+f(u)_{x}=0
$$

we use a conservative finite difference scheme

$$
u_{t}=-\frac{1}{\Delta x}\left(\hat{f}_{j+\frac{1}{2}}-\hat{f}_{j-\frac{1}{2}}\right)
$$

The term $\hat{f}_{j+\frac{1}{2}}=\hat{f}\left(u_{j-k}, \ldots, u_{j+l}\right)$ is the numerical $f u x$, and the points $\left\{x_{j-k}, \ldots, x_{j+l}\right\}$ constitute the stencil. To be a reasonable approximation, the numerical flux must be (at least) Lipschitz continuous and consistent with the physical flux $f$, i.e. $\hat{f}(u, \ldots, u)=f(u)$. The numerical flux determines the numerical method and its properties. Any differences between conservative numerical methods are a result of differences in the numerical flux.

Numerical methods for hyperbolic problems which feature discontinuities must satisfy non-linear stability requirements to ensure stability of the solution. Otherwise, when the derivative is approximated using points on both sides of the shock, oscillations arise. These oscillations at the shock location propagate to the smooth regions, destroying the stability of the solution. ENO (essentially non-oscillatory) schemes [11, 12] search for the locally smoothest stencil and use that stencil to calculate the numerical fluxes. The idea behind ENO schemes is stencil switching in order to eliminate oscillations. The stencil switching assures (asymptotically, at least) that the shock is not crossed, and thus linear stability is enough to ensure non-linear stability because to the left and to the right of the shock we have smooth regions, where linear stability is sufficient.

Weighted essentially non-oscillatory (WENO) schemes [16] use all the stencils that the ENO scheme considered, but assign a weight to each 
stencil. These weights approach the ENO weights near the discontinuity, but allow higher order differencing in the smooth region. To get an $r$ th-order ENO scheme, a total of $2 r-1$ points are examined to compute $\hat{f}_{j+\frac{1}{2}}$. Since the WENO scheme uses all the candidate stencils, a clever choice of weights [13] results in a WENO scheme which is of order $2 r-1$ in smooth regions. Thus, WENO methods are high-order finitedifference methods with an adaptive-stencil approach: at each point in space, they approximate the derivative with a high-order difference formula selected to prevent oscillations. WENO methods capture sharp gradients without smearing, and do not allow oscillations to appear and thus preserve the correct physical behavior by upwinding and stencil choosing. WENO schemes have proven useful in resolving the numerical solution of conservation laws with shocks $[13,16]$. However, these methods are also needed whenever sharp gradients are present, to prevent non-physical oscillations from appearing, propagating, and ultimately destroying the reliability of the numerical method.

We will be using the fifth-order WENO scheme. Since this scheme is described in detail in [13], we will not repeat the description of the algorithm here. We remark that, in the case of a system, we perform a characteristic decomposition and apply the scalar WENO scheme to each of the characteristic fields.

\section{GEGENBAUER POSTPROCESSING}

Given $2 N+1$ Fourier coefficients $\hat{f}_{k},-N \leqslant k \leqslant N$, of a continuous and periodic function $f(x)$, we can approximate the function using the classical Fourier sum

$$
f_{N}(x)=\sum_{k=-N}^{N} \hat{f}_{k} e^{i k \pi x}
$$

This approximation converges exponentially for any point in the domain if $f(x)$ and its periodic extension are analytic. However, if the function $f(x)$ is non-periodic or has a discontinuity, $f_{N}(x)$ does not approximate $f(x)$ well. In particular, away from the discontinuity (or boundary, in the case of a non-periodic function) the pointwise approximation error decays very slowly (as $O\left(\frac{1}{N}\right)$ ). Near the discontinuity (or boundary) there is an overshoot that does not diminish even with increasing $N$. These two features-the slow convergence away from the discontinuity and the overshoot at the discontinuity - are known as the "Gibbs phenomenon". Recent work [6-10] has shown that the Gibbs phenomenon can be completely removed, 
i.e. exponential accuracy recovered up to the discontinuity, by a Gegenbauer polynomial postprocessing procedure.

The idea of Gegenbauer polynomial postprocessing is that, rather than reconstruct the function naively by summing as in (1), we sum:

$$
f_{N}^{m}(x)=\sum_{k=0}^{m} \hat{g}_{k}^{\lambda} C_{k}^{\lambda}(\xi),
$$

where $\hat{g}_{k}^{\lambda}$ for $0 \leqslant k \leqslant m$ are called the Gegenbauer coefficients and are obtained from the Fourier partial sum $f_{N}(x)$, and $C_{k}^{\lambda}(x)$ are the Gegenbauer polynomials which are orthogonal over $x \in[-1,1]$ with the weight function $\left(1-x^{2}\right)^{\lambda-\frac{1}{2}}$. The Gegenbauer coefficients are defined by

$$
\hat{g}_{k}^{\lambda}=\frac{1}{h_{k}^{\lambda}} \int_{-1}^{1}\left(1-x^{2}\right)^{\lambda-\frac{1}{2}} f_{N}(x) C_{k}^{\lambda}(x) d x,
$$

where

$$
h_{k}^{\lambda}=\sqrt{\pi} \frac{\Gamma(k+2 \lambda)}{k ! \Gamma(2 \lambda)} \frac{\Gamma\left(\lambda+\frac{1}{2}\right)}{\Gamma(\lambda)(k+\lambda)} .
$$

We refer to this procedure as a postprocessing procedure because the Fourier partial sum (1) is used to calculate the Gegenbauer coefficient, which is then used in the summation.

The technique we use in the numerical examples takes $f_{N}\left(x_{j}\right)$ for $j=0, \ldots, 2 N$, the data from the WENO scheme. This function $f_{N}$ is not the usual Fourier projection, but rather the trigonometric interpolant. This data approximates the exact solution $f\left(x_{j}\right)$ for the points $x_{j}=-1+\frac{j}{N}$. We calculate the Fourier coefficients $\hat{f}_{k}$ where $k=-N, \ldots, N$. Although the Fourier coefficients are obtained from the entire domain $x \in(-1,1)$, we are interested in reconstructing the function in a subdomain of analyticity, $x \in(a, b)$. Thus,we define $x=\epsilon \xi+\delta$ where $\epsilon=\frac{b-a}{2}$ and $\delta=\frac{a+b}{2}$. The Gegenbauer polynomials $C_{l}^{\lambda}(\xi)$ are computed, and then the first $m+1$ Gegenbauer coefficients can be calculated:

$$
\begin{aligned}
g_{l}^{\lambda} & =\frac{1}{h_{l}^{\lambda}} \int_{-1}^{1}\left(1-\xi^{2}\right)^{\lambda-\frac{1}{2}} f_{N}(x(\xi)) C_{l}^{\lambda}(\xi) d \xi \\
& =\frac{1}{h_{l}^{\lambda}} \int_{-1}^{1}\left(1-\xi^{2}\right)^{\lambda-\frac{1}{2}} f_{N}(\epsilon \xi+\delta) C_{l}^{\lambda}(\xi) d \xi \\
& =\frac{1}{h_{l}^{\lambda}} \int_{-1}^{1}\left(1-\xi^{2}\right)^{\lambda-\frac{1}{2}} \sum_{k=-N}^{N} \hat{f}_{k} e^{i \pi k \epsilon \xi} e^{i \pi k \delta} C_{l}^{\lambda}(\xi) d \xi
\end{aligned}
$$




$$
\begin{aligned}
& =\sum_{k=-N}^{N} \hat{f}_{k} e^{i \pi k \delta} \frac{1}{h_{l}^{\lambda}} \int_{-1}^{1}\left(1-\xi^{2}\right)^{\lambda-\frac{1}{2}} e^{i \pi k \epsilon \xi} C_{l}^{\lambda}(\xi) d \xi \\
& =\sum_{k=-N}^{N} \hat{f}_{k} e^{i \pi k \delta} \Gamma(\lambda)\left(\frac{2}{\pi \epsilon k}\right)^{\lambda} i^{l}(l+\lambda) J_{l+\lambda}(\pi \epsilon k) .
\end{aligned}
$$

This last equality appears in [2] where $\Gamma$ is the Gamma function and $J_{p}$ is the order $p$ Bessel function.

While the error $\left|f-f_{N}\right|$ does not converge uniformly, the error from the postprocessed function $\left|f-f_{N}^{m}\right|$ does converge uniformly in every region where $f$ is smooth. The postprocessed error has been shown to decay exponentially with $N$, when $\lambda$ and $m$ are proportional to $N$ [610]. In our numerical experiments, where the underlying method is the fifth-order WENO scheme, we cannot hope for exponential convergence. However, we can hope to recover up to fifth-order accuracy away from the shock, or third-order near the shock (because the WENO scheme has third-order building blocks, which in smooth regions are combined in a way that gives fifth order), which was lost due to the discontinuity. As we show in Sec. 4, we do indeed recover design accuracy in smooth regions.

\section{NUMERICAL EXPERIMENTS}

For all our numerical experiments, we use results of a fifth-order point value WENO code with Roe building blocks, and third-order strong stability preserving Runge-Kutta time-stepping [18], for the quasi-onedimensional converging-diverging nozzle flow. The governing equations are the usual Euler system plus a source term:

$$
\left(\begin{array}{c}
\rho \\
\rho u \\
E
\end{array}\right)_{t}+\left(\begin{array}{c}
\rho u \\
P+\rho u^{2} \\
u(P+E)
\end{array}\right)_{x}=-\frac{A_{x}}{A}\left(\begin{array}{c}
\rho u \\
\rho u^{2} \\
u(P+E)
\end{array}\right),
$$

where $\rho, u, P$, and $E$ are the density, velocity, pressure, and total energy respectively, $A=A(x)$ is the cross area function of the nozzle and $A_{x}=\frac{d A}{d x}$. The shape of the nozzle is calculated by the requirement of linear distribution of Mach number from $M=0.8$ at the inlet to $M=1.8$ at the exit assuming the flow is isentropic and fully expanded. The equation of state is

$$
P=(\gamma-1)\left(E-\frac{1}{2} \rho u^{2}\right) .
$$

We compute the solution at steady-state by integrating in time until the residuals go down to machine zero. The exact solution is obtained, 
for the purpose of computing the errors, by a Newton iteration. The steady-state solution has a shock halfway across the domain. Although the shock location was known to us in this case, we used the edge detection method of Archibald, Gelb and Yoon [1] to locate it, and the results were identical. In general, if the shock location is unknown, it can be found using such an edge detection method. However, the important information is not, in fact, the shock location, but the intervals of smoothness. Thus, if the shock location is unclear, we can exclude a small portion of the interval to ensure that we are postprocessing over an interval of smoothness.

We separate the domain into two regions in space, the left of the shock and the right of the shock. In each of these regions the solution is analytic. The region to the right of the shock contains information that traveled through the shock, the left does not. As expected, the region to the left maintains high-order accuracy away from the shock, while in the region to the right of the shock, the error is only first-order accurate away from the shock and zeroth order accurate near the shock. For this particular example, design accuracy can be obtained even before postprocessing if we place the shock exactly in the middle between two gridpoints, but this is not always possible for general problems with unknown shock locations.

Table I shows the maximum norm errors for the density in the entire region to the right of the shock before and after postprocessing. We exclude only one gridpoint near the shock since, by conservation, the shock will corrupt the solution at that point. Thus, the domain in which we are measuring the error is actually getting closer and closer to the shock as $N$ increases, thus emulating uniform convergence. The maximum norm errors of the numerical solution before postprocessing do not decay when the grid is refined. After postprocessing the region to the right of

Table I. The Maximum Norm Errors from the Steady-State Computation, before and after Postprocessing. The Errors are Calculated in the Entire Region to the Right of the Shock, Up to One Gridpoint away from the Shock

\begin{tabular}{rlllll}
\hline \multicolumn{1}{c}{$n$} & Before error & $\lambda$ & $m$ & After error & Order \\
\hline 600 & $1.37 \times 10^{-3}$ & 3 & 3 & $8.16 \times 10^{-4}$ & \\
800 & $1.09 \times 10^{-3}$ & 3 & 4 & $3.33 \times 10^{-4}$ & 3.11 \\
1000 & $1.27 \times 10^{-3}$ & 4 & 5 & $1.66 \times 10^{-4}$ & 3.13 \\
1200 & $1.19 \times 10^{-3}$ & 5 & 6 & $8.19 \times 10^{-5}$ & 3.8 \\
1400 & $1.27 \times 10^{-3}$ & 6 & 7 & $4.09 \times 10^{-5}$ & 4.5 \\
1600 & $1.19 \times 10^{-3}$ & 6 & 8 & $1.72 \times 10^{-5}$ & 6.46 \\
1800 & $1.19 \times 10^{-3}$ & 7 & 9 & $8.95 \times 10^{-6}$ & 5.54 \\
\hline
\end{tabular}


Table II. The $l_{2}$ Norm Errors from the Steady-State Computation, before and after Postprocessing. The Errors are Calculated in the Entire Region to the Right of the Shock, Up to One Gridpoint away from the Shock

\begin{tabular}{rllllll}
\hline \multicolumn{1}{c}{$n$} & Before error & Order & $\lambda$ & $m$ & After error & Order \\
\hline 800 & $6.99 \times 10^{-5}$ & & 3 & 4 & $5.823 \times 10^{-5}$ & \\
1000 & $6.55 \times 10^{-5}$ & 0.29 & 4 & 5 & $2.605 \times 10^{-5}$ & 3.6 \\
1200 & $5.84 \times 10^{-5}$ & 0.63 & 5 & 6 & $1.313 \times 10^{-5}$ & 3.75 \\
1400 & $5.54 \times 10^{-5}$ & 0.34 & 6 & 7 & $6.169 \times 10^{-6}$ & 4.9 \\
1600 & $5.08 \times 10^{-5}$ & 0.65 & 6 & 8 & $3.968 \times 10^{-6}$ & 3.30 \\
1800 & $4.79 \times 10^{-5}$ & 0.50 & 7 & 9 & $2.528 \times 10^{-6}$ & 3.83 \\
\hline
\end{tabular}

the shock, high-order accuracy is recovered. Table II repeats this analysis for $l_{2}$ errors. The errors before postprocessing are half-order accurate. After postprocessing, the design order of accuracy (third-order near the shock) is recovered. Fig. 1 shows the exact density profile in the entire nozzle. Fig. 2 shows a close-up of the numerical density (before postprocessing) for 600,1000 , and 1600 points, to the right of the shock. This figure shows that the errors near the shock do not improve when the grid is refined. Fig. 3 shows the numerical density near the shock, before and after postprocessing which demonstrates how the oscillations are removed by postprocessing. Finally, Fig. 4 shows the logarithm of the errors before and after postprocessing in a region near the shock, and Fig. 5 shows the decay of the errors before and after postprocessing, as the grid is refined.

These numerical results demonstrate that the design order of accuracy was recovered right up to the shock after postprocessing. In this study, the dramatic improvements were most clear in the region closest to the shock. It is also important to note that we let $\lambda$ and $m$ vary linearly with $N$ (where $2 N+1$ is the number of Fourier coefficients), so that $\lambda \approx \frac{2 N}{250}$ and $m=\frac{2 N}{200}$. The truncation and regularization errors in the Gegenbauer postprocessing vary with $m$ and $\lambda$. Increasing these parameters with $N$ prevents the postprocessing error from overwhelming the discretization error in the postprocessed WENO solution.

\section{CONCLUDING REMARKS AND FUTURE WORK}

We have demonstrated through a numerical example the possibility of recovering the design high-order accuracy information up to the shock from WENO scheme solutions for one-dimensional non-linear hyperbolic systems, through the Gegenbauer postprocessor. More numerical tests and 


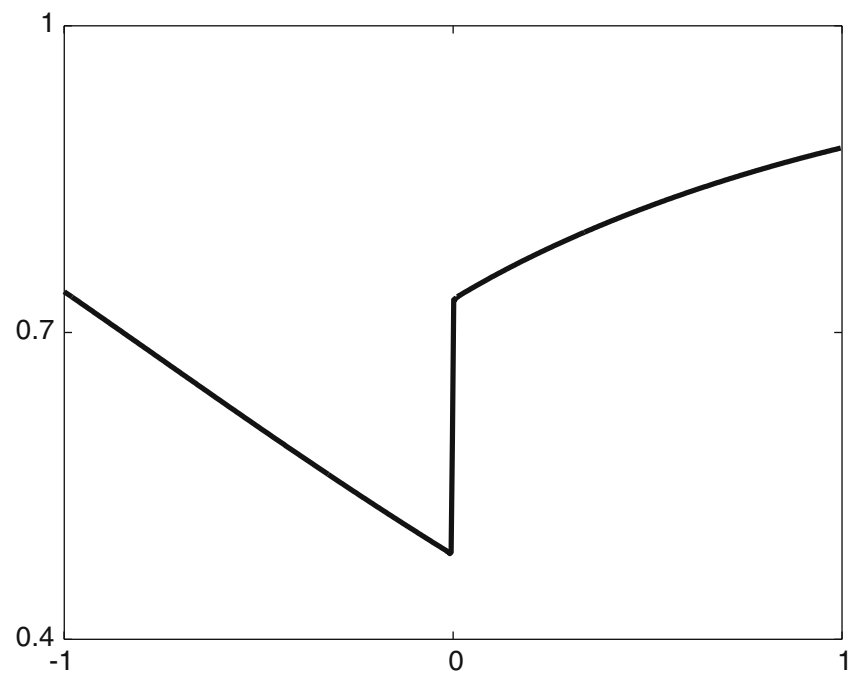

Fig. 1. Density profile over the entire region.

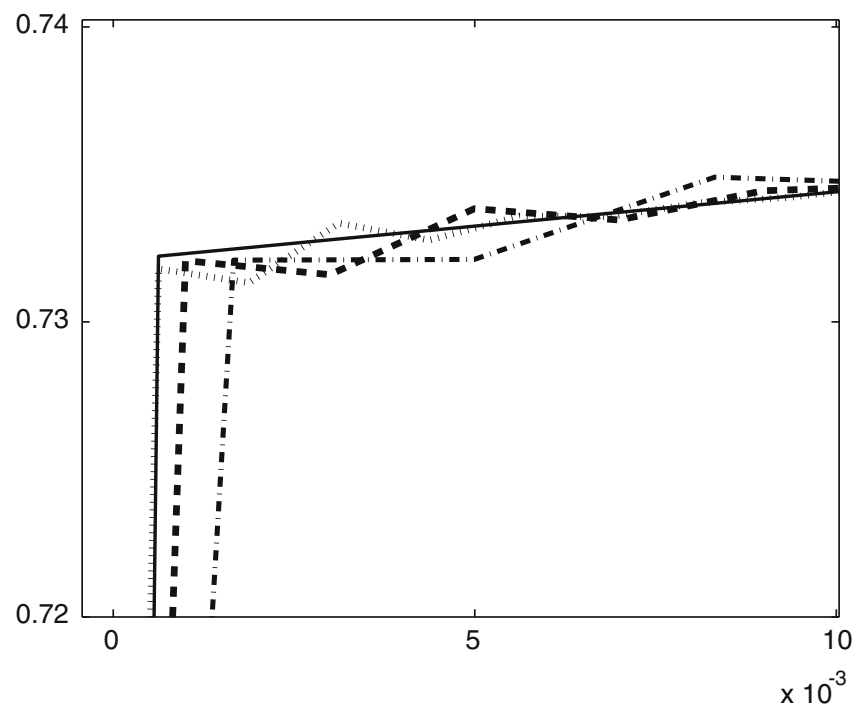

Fig. 2. Density profile close to the shock. The solid line is the exact solution, the dash-dot line $(2 N=600)$, dashed line $(2 N=1000)$ and dotted line $(2 N=1600)$ are the numerical solutions before postprocessing. 

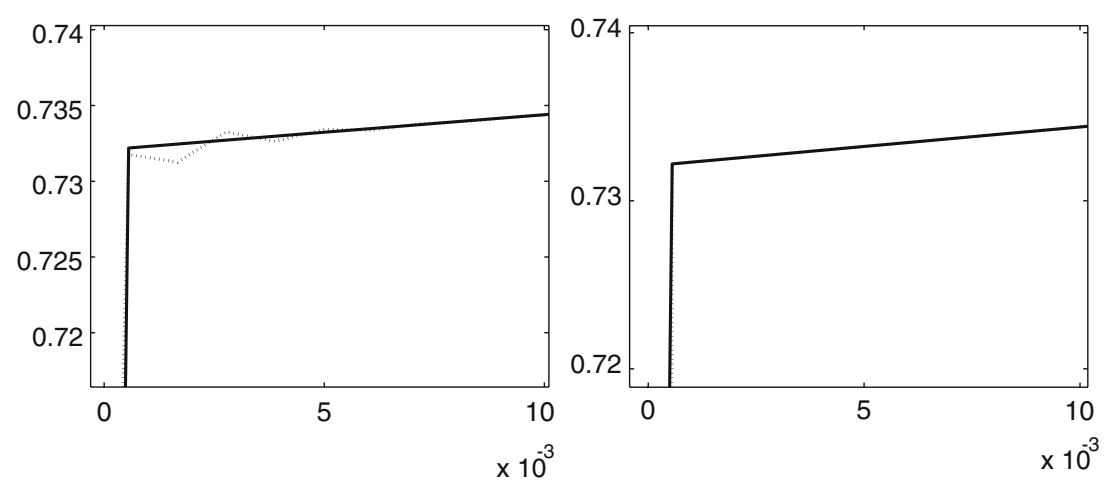

Fig. 3. Density profile close to the shock. The solid line is the exact solution, the dotted lines are the numerical solutions (for $2 N=1800$ ) before postprocessing (on left) and after postprocessing (right).
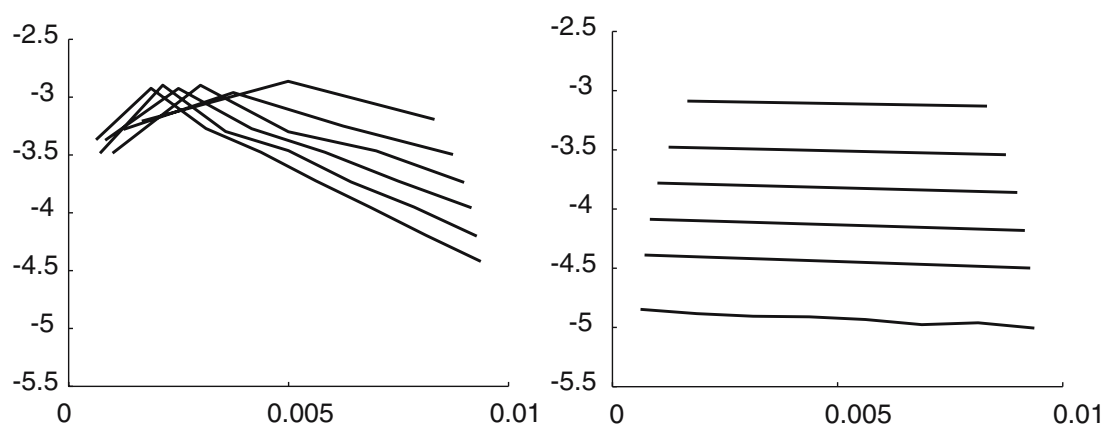

Fig. 4. Logarithm of the density errors, in a focused region to the right of the shock, before (left) and after (right) postprocessing for $2 N n=600,800,1000,1200,1400$, and 1600 points.
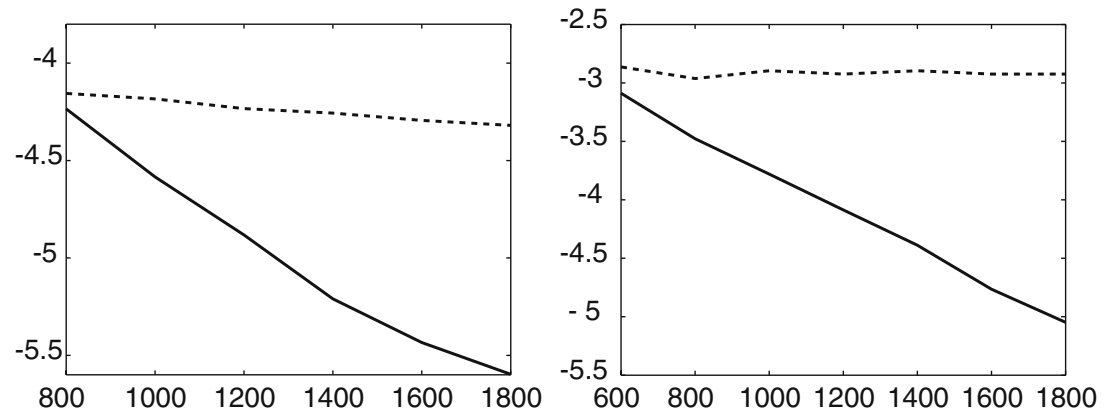

Fig. 5. Decay of the errors as the grid is refined. Left: logarithm of the $l_{2}$ errors before (dashed line) and after (solid line) postprocessing. Right: logarithm of the $l_{\infty}$ errors before (dashed line) and after (solid line) postprocessing. 
theoretical justifications beyond our preliminary numerical work are necessary to clearly address this issue. Further work is needed to generate robust Gegenbauer postprocessors which will improve the magnitude of the errors as they recover the order of accuracy. Clearly, the optimal choice of the parameters $m$ and $\lambda$ will also play a significant role in the implementation of the Gegenbauer postprocessor (see [4]). However, the most important future aspect will be the generalization to multiple dimensions and to cases in which the location of the discontinuity is unknown. This research will follow along the lines that the postprocessing for spectral and the spectral viscosity and superviscosity methods for multiple dimensions has been studied, and will utilize some of the cutting edge methods for detecting a discontinuity, such as the edge detection methods of Archibald et al. [1] and Gelb and Tadmor [5]. The effect of this study has been to show that WENO - and perhaps other finite difference - computations may be considered part of the family of methods that can be postprocessed to recover accuracy. Future work will also attempt to seek theoretical justification to these results, perhaps for specific cases at the beginning.

\section{ACKNOWLEDGMENTS}

Sigal Gottlieb was supported by NSF grant DMS 0106743. David Gottlieb was supported by AFOSR grant FA9550-05-1-0123 and DOE grant DE-FG02-98ER25346. Chi-Wang Shu was supported by ARO grant W911NF-04-1-0291 and NSF grant DMS-0510345. We also thank Rick Archibald and Anne Gelb for the use of their code.

\section{REFERENCES}

1. Archibald, R., Gelb, A., and Yoon, J. (2005). Polynomial fitting for edge detection in irregularly sampled signals and images. SIAM J. Numer. Anal. 43, 259-279.

2. Bateman, H. (1953). Higher Transcendental Functions, Vol. 2, McGraw-Hill, New York.

3. Casper, J., and Carpenter, M. (1998). Computational considerations for the simulation of shock induced sound. SIAM J. Sci. Comput. 19, 813-828.

4. Gelb, A. (2004). Parameter optimization and reduction of round off error for the Gegenbauer reconstruction method. J. Sci. Comput. 20, 433-459.

5. Gelb, A., and Tadmor, E. (2000). Enhanced spectral viscosity approximations for conservation laws. Appl. Numer. Math. 33, 3-21.

6. Gottlieb, D., Shu, C.-W., Solomonoff, A., and Vandeven, H. (1992). On the Gibbs phenomenon I: recovering exponential accuracy from the Fourier partial sum of a non-periodic analytic function. J. Comput. Appl. Math. 43, 81-98.

7. Gottlieb, D., and Shu, C.-W. (1996). On the Gibbs phenomenon III: recovering exponential accuracy in a sub-interval from a spectral partial sum of a piecewise analytic function. SIAM J. Numer. Anal. 33, 280-290. 
8. Gottlieb, D., and Shu, C.-W. (1995). On the Gibbs phenomenon IV: recovering exponential accuracy in a sub-interval from a Gegenbauer partial sum of a piecewise analytic function. Math. Comput. 64, 1081-1095.

9. Gottlieb, D., and Shu, C.-W. (1995). On the Gibbs phenomenon V: recovering exponential accuracy from collocation point values of a piecewise analytic function. Numerische Mathematik. 71. 511-526.

10. Gottlieb, D., and Shu, C.-W. (1997). On the Gibbs phenomenon and its resolution. SIAM Rev. 30, 644-668.

11. Harten, A., Engquist, B., Osher, S., and Chakravarthy, S. (1987). Uniformly high order essentially non-oscillatory schemes I. SIAM J. Numer. Anal. 24, 279-309.

12. Harten, A., Engquist, B., Osher, S., and Chakravarthy, S. (1987). Uniformly high order essentially non-oscillatory schemes III. J. Comput. Phys. 71, 231-303.

13. Jiang, G.-S., and Shu, C.-W. (1996). Efficient implementation of weighted ENO schemes. J. Comput. Phys. 126, 202-228.

14. Lax, P. D. (1978). Accuracy and resolution in the computation of solutions of linear and nonlinear equations. In De Boor, C., and Golub, G. (eds.), Recent Advances in Numerical Analysis, Academic Press, New York.

15. Lax, P., and Mock, M. (1978). The computation of discontinuous solutions of linear hyperbolic equations. Commun. Pure Appl. Math. 31, 423-430.

16. Liu, X-D., Osher, S., and Chan, T. (1994). Weighted essentially non-oscillatory schemes. J. Comput. Phys. 115, 200-212.

17. Majda, A., and Osher, S. (1977). Propagation of error into regions of smoothness for accurate difference approximations to hyperbolic equations. Commun. Pure Appl. Math. 30, 671-705.

18. Shu, C.-W., and Osher, S. (1988). Efficient implementation of essentially non-oscillatory shock-capturing schemes. J. Comput. Phys. 77, 439-471.

19. Shu, C.-W., and Wong, P. (1995). A note on the accuracy of spectral method applied to nonlinear conservation laws. J. Sci. Comput. 10, 357-369. 